\title{
The Art of Cheating When Drawing a Graph (Extended Abstract)
}

\author{
Martin Wattenberg \\ Visual Communication Lab \\ IBM Research \\ mwatten@us.ibm.com
}

\begin{abstract}
The prime directive of graph drawing is to depict a network faithfully and accurately. But sometimes it's better to cheat. I will discuss a series of examples - both my own work and that of others - that involve discarding information, distorting the data, encouraging visual clutter, or even adding random noise. The benefits of breaking the rules can range from the scientific to the artistic.
\end{abstract}

